\title{
Surgery should not be used as first-line treatment
}

\author{
Chippaux J-P (1)
}

(1) Research Institute for Development, Paris, France.

I was very surprised by the publication of the article by Fujioka et al. "Immediate radical fang mark ablation may allow treatment of Japanese viper bite without antivenom" published in The Journal of Venomous Animals and Toxins including Tropical Diseases, 2009, vol. 15, n. 1, p. 168-178.

The conclusions of this article could be confusing for practitioners managing snakebite envenomations that result in unacceptable risks for patients treated according to these "historical" and disputed techniques.

1. Therapeutic results from five patients, without any control, should not be conclusive and do not prove efficacy. One cannot affirm, by reading these observations, that the treatment was effective (however, this opinion is doubtful considering some of the scars!). Nevertheless, it is not possible to affirm that any other treatment could be more effective.

2. The reactogenicity of antivenoms has become rare and mild with the current highly purified antivenom immunoglobulins (about $5 \%$ of mild adverse effects and less than $0.1 \%$ of adverse reactions which could be considered worrying). If the authors continue to see frequent and/or severe adverse effects, it means that the antivenom employed by them presents poor quality, so that they should replace it or encourage the manufacturer to improve production methods. Furthermore, antivenom adverse effects are easily treated by accessible and cheap drugs. Practitioners' perspective should advance on this issue: current highly purified antivenoms are not considered dangerous anymore.

3. The risks of hemorrhage from surgical ablations after viper envenomation are extremely high and add a morbidity factor to the envenomation severity. The risks of infections, even of nosocomial infections, are not negligible... 
1. The required delay to implement surgical ablation (at least one hour in the cases presented by the authors) allowed the diffusion of venom and its systemic consequences.

2. In my personal experience, early surgical ablations of necrotic zones often result in relapses inducing new surgical interventions during the following days. I argued that ablation is not always radical and the diffusion of venom components may require various steps. I deduced from these observations that it was more advisable to wait for the stabilization of the necrotic lesions, ensuring local asepsis and waiting for coagulation restoration, which can take several days (Chippaux J-P, "Les complications locales des morsures de serpents", Médecine Tropicale, 1982, vol. 42, p. 177-183).

Studies are in progress to propose safe and effective alternatives to surgical ablation. Specific metalloprotease inhibitors can be applied locally to neutralize the venom components that remained in situ (Gutiérrez et al., "Trends in snakebite envenomation therapy: scientific, technological and public health considerations", Current Pharmaceutical Design, 2007, vol. 13, n. 28, p. 2935-2950). Moreover, with the improvement of antivenoms, now we can expect a higher efficacy against necrotic lesions.

Definitively, I am afraid that surgery is not the best manner to treat and cure envenomations...

\section{CORRESPONDENCE TO:}

JEAN-PHILIPPE CHIPPAUX, Institut de Recherche pour le Développement, IRD, Laboratoire de Parasitologie, Facultée de Pharmacie Paris V, 4 Avenue de l'Observatoire, 75006 Paris, France. Phone: +33 153731507. Fax: +33 153739617. Email: jean-philippe.chippaux@ird.fr. 